\title{
To Give or Not to Give? Lessons from the Arginine Paradox
}

\author{
Francesco Saverio Dioguardi \\ Department of Internal Medicine, University of Milan, Milan, Italy
}

\section{Key Words}

Amino acids $\cdot$ Arginine $\cdot$ Citric acid cycle $\cdot$ Glutamine $\cdot$ Malnutrition $\cdot$ Nitric oxide $\cdot$

Nitric oxide synthases

\begin{abstract}
Arginine is one of the 20 amino acids (AA) found in proteins and synthesized by human cells. However, arginine is also the substrate for a series of reactions leading to the synthesis of other $A A$ and is an obligatory substrate for two enzymes with diverging actions, arginases and nitric oxide synthases (NOS), giving origin to urea and NO, respectively. NO is a very potent vasodilator when produced by endothelial NOS (eNOS). The 'arginine paradox' is the fact that, despite intracellular physiological concentration of arginine being several hundred micromoles per liter, far exceeding the $\sim 5 \mu \mathrm{M} \mathrm{K}$ of eNOS, the acute provision of exogenous arginine still increases NO production. Clinically, an additional paradox is that the largest controlled study on chronic oral arginine supplementation in patients after myocardial infarction had to be interrupted for excess mortality in treated patients. Expression and activity of arginases, which produce urea and divert arginine from NOS, are positively related to exogenous arginine supplementation. Therefore, the more arginine is introduced, the more it is destroyed, eventually leading to impaired NO production. In this review, conditions influencing the low arginine concentrations found in plasma will be reviewed, revising the paradigm that simple replenishment of what is lacking will always produce beneficial consequences. Copyright $\odot 2011$ S. Karger AG, Basel
\end{abstract}

\section{Arginine, Nitric Oxide and Related Paradoxes}

Arginine is one of the 20 amino acids (AA) necessary for protein synthesis and coded by DNA. Plasma arginine concentrations are therefore maintained mostly by protein catabolism $(\approx 85 \%)$ [1] or by synthesis from other AA. Of the total synthesis, de novo synthesis ac- 
counts for $<15 \%$, while $60 \%$ of arginine is derived from citrulline [2]. Arginine metabolism may give rise to several other AA and molecules indispensable for life (fig. 1). Exogenous arginine is largely destroyed $(\approx 40 \%)$ during absorption by the gut itself. Much attention was given to arginine availability due to the discovery in the 1980s that 'endothelium-dependent relaxing factor' is a gas, nitric oxide (NO), produced from arginine by endothelial NO synthases (eNOS) [3] in a reaction that gives also rise to citrulline. In endothelial cells, eNOS and the two sequential enzymes argininosuccinate lyase (ASL) and argininosuccinate synthase (ASsynth), necessary for recycling citrulline to arginine, are co-localized in the caveolae [4], a fraction of cell membranes that provides an efficient environment for maintaining arginine available to eNOS. The cytoplasm of endothelial cells has a concentration of arginine largely saturating eNOS, and yet the acute introduction of exogenous arginine elicits an increase in NO production, a puzzling finding known as 'the arginine paradox' [5]. Another puzzling finding is the peculiar regulation of arginine transport and its effects on NO. The transport of arginine into cells is mediated by the cationic AA transporter 1 (CAT1). Overexpression of CAT1 enhances arginine uptake sixfold, and NO production twofold. The presence of AA competing with arginine decreases arginine uptake, but not NO production [4]. Moreover, citrulline succeeds in stimulating NO production even in a medium containing saturating levels of arginine, and extracellular citrulline does not influence intracellular arginine levels. Therefore, NO production depends mostly on the efficient recycling of arginine-derived citrulline back to arginine, and not so much on exogenous arginine supply. Thus, the presence of a 'micro-environment', where arginine metabolism and recycling are independently regulated and only partially in balance with plasma arginine concentrations, is the most consistent explanation for the endothelial arginine paradox [6].

An even more puzzling paradox is that long-term arginine supplementation is ineffective in improving eNOS activity $[7,8]$. Even worse, exogenous arginine increased mortality when arginine was given to promote vasodilation in patients after a myocardial infarction [9]. We will now analyze these findings in more detail.

\section{Endothelial Vasomotion and the Exogenous Arginine Paradox}

To understand the clinical paradox of arginine, i.e. the fact that the much needed arginine is ineffective or actually detrimental when supplemented chronically, we have to focus on another pathway of the complex arginine metabolism (fig. 1), which is controlled by the ubiquitous enzymes arginases (in two types, 1 and 2), which compete with NOS for arginine as a substrate. The effect of both arginases is to catalyze the cleavage of urea from arginine, thus forming ornithine. Urea controls osmolarity and water content of plasma and cells. The other product of the reaction, ornithine, may be recycled to citrulline and also synthesized de novo by the liver from glutamine, and then transformed by the gut into citrulline. The kidneys provide the further recycling of citrulline to arginine, finally released into the plasma [8]. Arginase 1 (ARG 1) is a cytosolic enzyme, mostly expressed in the liver and red blood cells. Deletion of the ARG 1 gene, as occasionally found in humans, is incompatible with prolonged life, and the accompanying hyperargininemia is associated with a several-fold increase in the activity of the mitochondrial arginase (ARG 2), which accounts for the persistent ureagenesis in those patients [10]. ARG 2 is widely expressed, mostly in the kidneys, gut and brain. ARG 2 overexpression plays a critical role in the pathophysiology of cholesterolmediated endothelial dysfunction [11]. Arginases and NOS compete for arginine, and - under any conditions - arginase activity exceeds NOS activity at all NOS/arginase molar ratios [12]. Moreover, although the $\mathrm{K}_{\mathrm{M}}$ of arginases is 100 -fold higher than that of NOS, the enzymes compete for arginine because the maximal catalytic rate of arginases is more than 
1,000 times higher than that of NOS $[2,13]$. Therefore, increased expression and/or activity of ARG has a deep impact on NOS efficiency. As an example, arginase activity is increased in type 2 diabetic subjects with impaired NOS activity, and such impairment correlates with the degree of hyperglycemia and is reduced by insulin [14].

\section{Arginine Controls Arginase Expression and May Be Used as a Fuel}

Exogenous arginine induces both ARG 1 and ARG 2 expression [10, 15]. Thus, eventually, the more arginine is introduced, the more it is destroyed. Accordingly, it has been demonstrated that in the liver, arginase activities strongly correlate with arginine concentrations, and inhibition of arginases peculiarly enhances the activity of the cytokine-inducible isoform of NOS (iNOS), which, in turn, activates cyclo-oxygenase-2 and inflammation [12].

Increased arginase activity would also negatively influence eNOS efficiency in producing NO, as observed in hypertensive humans where exogenous arginine is ineffective in improving vasodilation [16].

Arginine metabolism by arginases may have other inhibitory effects on eNOS activity and NO production because urea produced by arginases is a potent inhibitor of ASL [17], one of the enzymes recycling citrulline to arginine, which colocalizes with eNOS. Also, ornithine produced from arginine when urea is cleaved by arginases (fig. 1) is a potent inhibitor of ARG 1, but a poor inhibitor of ARG 2 [18].

Additionally, arginine is a potent secretagogue for glucagon, which induces arginase in liver perivenous cells and the catabolism of arginine to ornithine [19], but also antagonizes insulin and causes insulin resistance [20]. This is important because insulin resistance increases the expression of glutaminase in liver periportal cells, thus promoting the wasting of glutamine, which is necessary for recycling ornithine to citrulline (fig. 1). Dietary glutamate and glutamine are nearly totally oxidized by small-intestine epithelial cells during absorption and are actively taken up from the blood stream when synthesized elsewhere because they are the most important fuel for the intestinal tissues [21, 22]. By using isotopically labeled molecules, it was found that the amino group of glutamine labels the three nitrogens of citrulline almost equally; only a nonspecific incorporation of carbon atoms from glutamine oxidation into carbamoyl phosphate - necessary for citrulline synthesis - has been observed [23]. Therefore, glutamine is synthesized from glutamate, which in turn derives from the citric acid cycle through the export of $\alpha$-ketoglutarate, a most important precursor of oxaloacetate.

The availability of glutamate, as that of aspartic acid (aspartate) necessary for aspartate synthase to start the recycling of citrulline to arginine, depends on the entry of glucose-derived pyruvate into the mitochondria. As described elsewhere [24], the occurrence of insulin resistance triggers a prevalence of fatty-acid $\beta$-oxidation in providing acetate for citrate synthesis. The prevalence of $\beta$-oxidation in providing acetate is accompanied by an inhibition of pyruvate dehydrogenase, preventing the entry into mitochondria of acetate derived from pyruvate and the entire glucose oxidation. Also, variations of the balances of NAD/NADH and NADP/NADPH in the cytoplasm due to changes in $\beta$-oxidation increase the drive of pyruvate towards oxaloacetate and then to citrate and fatty-acid synthesis, explaining the paradox that steatosis is present in cells where energy production is controlled by $\beta$-oxidation in conditions of chronic insulin resistance.

Intermediates of the citric acid cycle are necessary for the synthesis of nonessential AA, such as glutamic acid, glutamine and arginine, and for this purpose such intermediates have to be exported from the mitochondria to the cytoplasm. When export is possible, the citric acid cycle is defined as anaplerotic, in contrast with a cataplerotic cycle, which occurs when 
export of intermediates is insufficient to maintain an adequate synthesis of nonessential AA, which is the case when aspartate and glutamate are no longer produced sufficiently. Therefore, export of intermediates of the cycle is indispensable for anabolic purposes, but also subtracts intermediates that would be recycled to the oxaloacetate, indispensable for condensation with acetate and for maintaining the cycle fully working by forming new citrate. Insufficient oxaloacetate availability into the cycle is followed both by a reduction of energy production and, subsequently, by a reduction of the synthesis of nonessential AA from intermediates of the cycle. This process, however, also triggers the entry of AA into mitochondria to refuel the cycle of oxaloacetate precursors, mostly at the level of succinate or $\alpha$-ketoglutarate. Such phenomena explain the pathophysiological mechanism underlying the sarcopenia/cachexia syndrome occurring in all conditions of peripheral insulin resistance.

It should be kept in mind that the prevalence of $\beta$-oxidation is unfavorable for the production of energy not only because for any acetate produced from fatty acids the amount of ATP produced is $25 \%$ less than for any acetate produced from glucose, but also because the citric acid cycle is 'choked' by the lack of oxaloacetate in amounts sufficient to maintain the efficiency of the citric acid cycle in both exporting intermediates (anaplerotic citric acid cycle) or maintaining a full efficiency for citrate and ATP synthesis.

Proof of this concept is that trimetazidine, an inhibitor of $\beta$-oxidation, improves ventricular ejection fraction in patients with insulin resistance in whom $\beta$-oxidation prevails [25]. Glutamine and arginine are excellent substrates for the citric acid cycle because both may be efficient precursors of $\alpha$-ketoglutarate, and this explains why, in demanding metabolic conditions such as in sepsis, both these AA can be found substantially decreased in plasma [26]. Moreover, elevated concentrations of glutamine increase ASsynth expression and activity in intestinal cells, similarly to interleukin (IL)-1 $\beta$, but the simultaneous presence of glutamine and IL-1 suppresses ASsynth expression and activity [27]. In contrast, glutamine inhibits arginine synthesis in endothelial cells by directly decreasing ASsynth activity and also through a competitive inhibition of citrulline uptake [28].

In summary, oral arginine supplementation not only increases arginine disposal through urea synthesis, but also promotes insulin resistance, a detrimental condition that reduces the availability of components necessary for both de novo and recycling synthesis. Therefore, indications for glutamine supplementation, out of the scope of this review, should be carefully revised [29].

\section{Interactions between iNOS and eNOS and Control of eNOS Expression}

Because arginine is necessary for iNOS to produce NO for bactericidal purposes in macrophages and is also used by $\mathrm{T}$ lymphocytes for their own function and proliferation, arginine availability is strictly linked to the immune response. At variance with rodents, human macrophages express little arginase or iNOS unless activated [30], but their production of $\mathrm{NO}$ is indispensable for the defense against bacteria, as shown by the poor outcome of patients with sepsis and low plasma arginine [31]. Inducible (i)NOS is not expressed constitutively by most cells, but is highly inducible by bacterial endotoxins and inflammatory cytokines. Unlike eNOS, iNOS are primarily cytosolic. Activation of macrophages through tolllike receptor signaling pathways is an important component of the immune defense, but some pathogens, such as mycobacteria, use this pathway to increase the expression of ARG 1 , reduce arginine availability and create conditions of resistance to infections [32]. Changes in the immune response may either be due to excessive activation, as observed in asthma, or defective activation, as in sepsis. However, NO production by NOS generates $\mathrm{N}_{\mathrm{G}}$-hydroxylL-arginine (NOHA), which is a potent natural inhibitor of arginase, reducing arginine avail- 


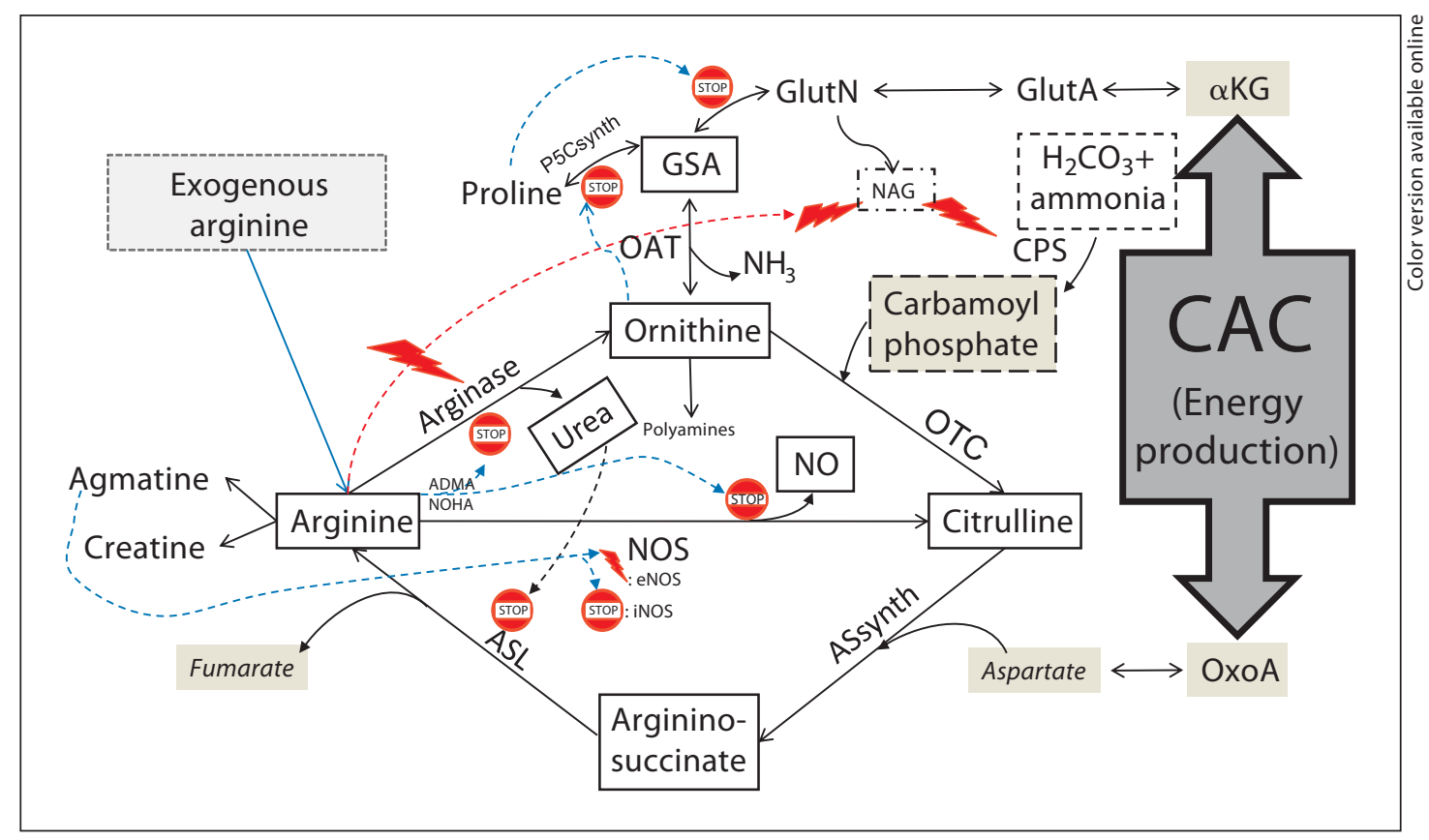

Fig. 1. A schematic representation of urea (arginine to ornithine-citrulline cycle), of citrulline-arginine recycling and of their relationship with the citric acid cycle (CAC). The CAC is the source of $\alpha$-ketoglutarate $(\alpha K G)$ and aspartate, and those two molecules are the link between the CAC and the urea and citrulline-arginine cycles. Aspartate is necessary for starting the recycling of citrulline to arginine by ASsynth. Arginine induces arginase expression activity (flash sign) and activates N-acetylglutamate (NAG) synthase, an allosteric activator indispensable for the synthesis of carbamoyl phosphate (CPS) and the action of ornithine transcarbamylase action. NAG synthesis depends on the availability of glutamine (GlutN), which is synthesized de novo from $\alpha \mathrm{KG}$ exported from the CAC, via glutamate synthesis (GlutA). Urea produced by arginases is a potent inhibitor of ASL. Ornithine derived from arginase action is both the substrate and the product for the synthesis or metabolism of proline and glutamine, and glutamic semialdehyde (GSA) is the intermediate of those reactions, mediated by ornithine aminotransferase (OAT), the enzyme exchanging one amino group between glutamate and $\alpha$ KG. Proline inhibits GSA synthesis by negative feedback on $\Delta^{2}$-pyrrolidine-5-carboxylate synthase (P5Csynth). Ornithine inhibits P5Csynth and proline synthesis from GSA. Agmatine increases eNOS and decreases inducible NOS activity. Arginine metabolism by NOS and protein catabolism produces variable amounts of physiological arginine competitors with inhibitory properties, here represented as NOHA and asymmetric dimethylarginine (ADMA). The bar signal at the end of dotted lines indicates inhibition. The flash sign inserted on dotted lines indicates expression and/or activation.

ability for urea synthesis. By this mechanism, elevated concentrations of NOHA improve arginine availability for NO synthesis. In turn, agmatine, generated from arginine (fig. 1), is a weak inhibitor of NOS but decreases the expression of iNOS and can increase endothelial NO production by binding to a cell surface imidazoline receptor and increasing cytosolic calcium [33]. Furthermore, the assembly of iNOS depends on arginine presence for dimerization, and the incomplete dimerization when arginase activity is prevalent would mean incomplete iNOS coupling and the presence of inactive enzymes [34]. The reader is referred to a comprehensive review on the synthesis and metabolism of endogenous arginine analogues with inhibitory activity on NOS and arginases other than NOHA [35].

To further complicate the picture, while the two arginase isoforms (I and II) are induced by T-helper- 2 regulatory cytokines (IL-4, IL-10, IL-13), TGF- $\beta$, and prostaglandin $\mathrm{E}_{2}$, the 


\section{Journal of \\ Nutrigenetics \\ Nutrigenomics}

Table 1. Cluster of AA tailored to human needs (CATHON; proportions are based on 100 $\mathrm{mg}$, L-forms)

\begin{tabular}{l|l}
\hline J Nutrigenet Nutrigenomics 2011;4:90-98 \\
\hline $\begin{array}{l}\text { DOl: 10.1159/000327777 } \\
\text { Published online: May 28, } 2011\end{array}$ & $\odot 2011$ S. Karger AG, Basel \\
\hline Dioguardi: Arginine: To Give or Not to Give?
\end{tabular}

$\begin{array}{lc}\text { Leucine } & 31.25 \mathrm{mg} \\ \text { Lysine } & 16.25 \mathrm{mg} \\ \text { Isoleucine } & 15.625 \mathrm{mg} \\ \text { Valine } & 15.625 \mathrm{mg} \\ \text { Threonine } & 8.75 \mathrm{mg} \\ \text { Cystine } & 3.75 \mathrm{mg} \\ \text { Histidine } & 3.75 \mathrm{mg} \\ \text { Phenyl-alanine } & 2.5 \mathrm{mg} \\ \text { Methionine } & 1.25 \mathrm{mg} \\ \text { Tyrosine } & 0.75 \mathrm{mg} \\ \text { Tryptophan } & 0.5 \mathrm{mg}\end{array}$

This formulation has peculiarities in that it contains two nonessential AA. Tyrosine is provided because it is an essential AA for all tissues apart from the liver. Cystine is contained in a peculiar molar ratio to methionine to meet sulfur AA requirements, minimizing the synthesis of homocysteine, the intermediate between methionine and cysteine.

expression of both NOS and of arginases may be induced by endotoxins and T-helper-1 cytokines (IL-1, TNF- $\alpha$, interferon- $\gamma$ ). It should also be remembered that ornithine is an important precursor both of polyamines needed for cell proliferation and of proline needed for collagen synthesis and wound healing. Therefore, T-helper-1 cells induce iNOS, while the induction of arginase by T-helper-2 cells suppresses iNOS production. This complex interregulation is further controlled by arginine availability [36]. There must be some kind of competition for arginine between iNOS and eNOS, since, in a model of allergic asthma, the overexpression of eNOS in lung endothelial cells results in both increased eNOS activity and NO production, and after sensitization with ovalbumin thoracic lymph node cells produced significantly less cytokines, with a 50\% reduction in the number of lymphocytes and eosinophils in the lung lavage fluid and an abolishment of airway methacholine hyperresponsiveness. The hyperresponsiveness of airways in asthma is associated with enhanced iNOS expression and activity of T cells, with increased levels of exhaled NO. The balance between the prevailing NO production by iNOS and the bronchodilator (by smooth muscle relaxation) NO production by eNOS may be a key to understanding asthma [37].

Our group has shown that aging increases iNOS and decreases eNOS expression in the kidneys. We also showed that supplementation with a cluster of essential AA, in specific stoichiometric ratios tailored for mammalian (hence, human) needs (CATHON ${ }^{\mathrm{TM}}$; table 1), is effective in reversing the balance among iNOS and eNOS, with improved expression of eNOS, increased mitochondrial biogenesis and cytochrome $\mathrm{C}$ oxidase activity, and parallel reduction of glomerular iNOS expression and fibrosis [38]. Similar effects had previously been observed in skeletal and myocardial muscles [39]. Even more interestingly, we have shown that the long-term supplementation with the same formulation increases mitochondrial biogenesis and upregulates gene systems for the defense against reactive oxygen species (ROS) and longevity. This was related to both increased expression of peroxisome proliferator-activated receptor- $\gamma$ coactivator- $1 \alpha$ (PGC-1 $\alpha$ ) and of sirtuin-1 (SIRT-1) expression and mammalian target of rapamycin (mTOR) signaling. Thus, we observed that eNOS expression and mitochondrial biogenesis are strictly linked [40]. The CATHON formulation does not contain arginine and yet proved to be extremely efficient in promoting improved physical activity both in diabetic patients with chronic heart failure [41] and patients with chronic obstructive pulmonary disease, where an impressive improvement in blood $\mathrm{PO}_{2}$ was doc- 
umented [42]. Interestingly, a 'side effect' of CATHON administration is improved insulin sensitivity, as shown by long-term supplementation studies in the elderly [43] and, more recently, in extremely severe intensive care unit patients [44]. CATHON enhances insulin action thanks to enhanced mTOR activity [45], with important clinical implications [22].

As discussed above, insulin resistance conditions are characterized by a citric acid cycle unable to export intermediates and consuming excessive amounts of AA for energy production, as shown in chronic heart failure patients submitted to light exercise [46]. In such conditions, as in any condition of unbalanced protein-calorie introduction, far more elevated amounts of essential AA than those usually contained in alimentary proteins in standard diets have to be provided to maintain the integrity of metabolism [47]. Moreover, an increased supplementation of essential AA not only improves energy production even in the most demanding conditions [48], but also improves the synthesis of any non-essential AA, replenishing the citric acid cycle and restoring the anaplerotic export of those intermediates necessary for maintaining the ubiquitous synthesis of any AA, glutamate and arginine included. In my opinion, this is the main reason why immune response is improved in intensive care patients [42] and long-term-care acquired infections are reduced in elderly patients receiving CATHON supplementation [49].

\section{Concluding Remarks}

Arginine metabolism is very complex, and equally complex and highly interregulated is the availability of arginine for the various metabolic pathways in which this AA is involved. Exogenous arginine supplementation should be avoided, because it does not resolve the reasons underlying its excess consumption or blunted synthesis, and, on the contrary, may actually worsen them. The usual paradigm of clinical nutrition, assuming that what is lacking should be provided, may here prove to be wrong. A better alternative is the provision of sufficient amounts of balanced formulations of essential AA, because these would better maintain and restore concentrations and metabolic pathways for the synthesis of this non-essential AA, and of its precursors and derivatives. Balanced formulations of essential AA are also the key to promoting gene expression of elements such as mTOR, PGC- $1 \alpha$, SIRT-1, and eNOS, consistent with the best possible metabolic performances in any physiological or pathological condition and at any age.

\section{References}

1 Morris SM Jr: Arginine: beyond protein. Am J Clin Nutr 2006;83(suppl):508S-512S.

2 Wu G, Morris SM Jr: Arginine metabolism: nitric oxide and beyond. Biochem J 1998;336:1-17.

-3 Ignarro LJ, Buga GM, Wood KS, Byrns RE, Chaudhuri G: Endothelium-derived relaxing factor produced and released from artery and vein is nitric oxide. Proc Natl Acad Sci USA 1987;84:9265-9269.

-4 Li C, Huang W, Harris MB, Goolsky JM, Venema RC: Interaction of endothelial nitric oxide synthase with the CAT-1 arginine transporter enhances NO release by a mechanism not involving arginine transport. Biochem J 2005;386: 567-574.

5 Kurz S, Harrison DG: Insulin and the arginine paradox. J Clin Invest 1997;99:369-370.

-6 Flam BR, Eichler DC, Solomonson LP: Endothelial nitric oxide production is tightly coupled to the citrulline-NO cycle. Nitric Oxide 2007;17:115-121.

7 Chin-Dusting JPF, Alexander CT, Arnold P, Hodgson WC, Lux AS, Jennings GLR: Effects of in vivo and in vitro Larginine supplementation on healthy human vessels. J Cardiovasc Pharmacol 1996;28:158-166.

8 Van de Poll MCG, Siroen MPC, van Leeuwen PAM, Soeters PB, Melis GC, Boelens PG, Deutz NEP, Dejong CHC: Interorgan amino acid exchange in humans: consequences for arginine and citrulline metabolism. Am J Clin Nutr 2007; $85: 167-172$. 


\section{Nutrigenetics \\ Nutrigenomics}

\begin{tabular}{l|l}
\hline J Nutrigenet Nutrigenomics 2011;4:90-98 \\
\hline DOI: 10.1159/000327777 & @ 2011 S. Karger AG, Basel \\
Published online: May 28, 2011 & \\
\hline
\end{tabular}

Dioguardi: Arginine: To Give or Not to Give?

-9 Schulman SP, Becker LC, Kass DA, Champion HC, Terrin ML, Forman S, Ernst KV, Kelemen MD, Townsend SN, Capriotti A, Hare JM, Gerstenblith G: L-Arginine therapy in acute myocardial infarction. The Vascular Interaction with Age in Myocardial Infarction (VINTAGE MI) randomized trial. JAMA 2006;295:58-64.

10 Grody WW, Argyle C, Kern RM, Dizikes DJ, Spector EB, Strickland AD, Klein D, Cederbaum SD: Differential expression of the two arginase genes in hyperargininemia. Enzymatic, pathologic, and molecular analysis. J Clin Invest 1989; 83:602-609.

11 Vanoutte PM: Arginine and arginase. Endothelial NO synthase double crossed? Circ Res 2008;102:866-868.

12 Santhanam L, Christianson DW, Nyhan D, Berkowitz DE: Arginase and vascular aging. J Appl Physiol 2008;105: $1632-1642$

13 Topal G, Brunet A, Walch L, Boucher JL, David-Dufilho M: Mitochondrial arginase II modulates nitric-oxide synthesis through non freely exchangeable L-arginine pools in human endothelial cells. J Pharmacol Exp Ther 2006;318: 1368-1374.

14 Kashyap SR, Park YM, Lara A, De Fronzo RA, Zhang R: Insulin reduces plasma arginase activity in type 2 diabetic patients. Diabetes Care 2008;31:134-139.

15 Mori M, Gotoh T, Nagasaki A, Takiguchi M, Sonoki T: Regulation of urea cycle enzyme genes in nitric oxide synthesis. J Inher Metab Dis 1998;21:59-71.

16 Holowatz LA, Kenney WL: Upregulation of arginase activity contributes to attenuated reflex cutaneous vasodilation in hypertensive humans. J Physiol 2007;581:863-872.

17 Menhyart J, Grof J: Urea as a selective inhibitor of argininosuccinate lyase. Eur J Biochem 1977;75:405-409.

18 Colleluori DM, Morris SM, Ash DE: Expression, purification, and characterization of human type 2 arginase. Arch Biochem Biophys 2006;389:135-143.

19 O'Sullivan D, Brosnan JT, Brosnan ME: Catabolism of arginine and ornithine in the perfused rat liver: effect of dietary protein and of glucagon. Am J Physiol Endocrinol Metab 2000;278:E516-E521.

-20 De Castro Barbosa T, Poyares LL, Machado UF, Nunes MT: Chronic oral administration of arginine induces GH gene expression and insulin resistance. Life Sci 2006;79:1444-1449.

-21 Blachier F, Boutry C, Bos C, Tomé D: Metabolism and functions of L-glutamate in the epithelial cells of the small and large intestines. Am J Clin Nutr 2009;90:814S-821S.

22 Newsholme P, Procopio J, Ramos Lima MM, Pithon-Curi TC, Curi R: Glutamine and glutamate - their central role in cell metabolism and function. Cell Biochem Funct 2003;21:1-9.

23 Marini JC, Didelija IC, Castillo L, Lee B: Glutamine: precursor or nitrogen donor for citrulline synthesis? Am J Physiol Endocrinol Metab 2010;299:E69-E79.

24 Dioguardi FS: Wasting and the substrate to energy controlled pathway: a role for insulin resistance and amino acids. Am J Cardiol 2004;93:6A-12A.

25 Neubauer S: The failing heart: an engine out of fuel. N Engl J Med 2007;356:1140-1151.

26 Luiking YC, Deutz NE: Exogenous arginine in sepsis. Crit Care Med 2007;35:S557-S563.

27 Brasse-Lagnel C, Lavoinne A, Loeber D, Fairand A, Bôle-Feysot C, Deniel N, Husson A: Glutamine and interleukin$1 \beta$ interact at the level of $\mathrm{Sp} 1$ and nuclear factor- $\kappa \mathrm{B}$ to regulate argininosuccinate synthetase gene expression. FEBS J 2007;274:5250-5262.

28 Wu G, Meininger CJ: Regulation of L-arginine synthesis from L-citrulline by L-glutamine in endothelial cells. Am J Physiol 1993;265:H1965-H1971.

29 Coëffier M, Déchelotte P: Combined infusion of glutamine and arginine: does it make sense? Curr Opin Clin Nutr Metab Care 2010;13:70-74.

- 30 Weinberg JB, Misukonis MA, Shami PJ, Mason SN, Sauls DL, Dittman WA, Wood ER, Smith GK, McDonald B, Bachus KE, Haney AF, Granger DL: Human mononuclear phagocyte inducible nitric oxide synthase (iNOS): analysis of iNOS mRNA, iNOS protein, biopterin, and nitric oxide production by blood monocytes and peritoneal macrophages. Blood 1995;86:1184-1195.

-31 Kao CC, Bandi V, Guntupalli KK, Wu M, Castillo L, Jahoor F: Arginine, citrulline and nitric oxide metabolism in sepsis. Clin Sci 2009;117:23-30.

32 Morris SM Jr: Arginine: master and commander in innate immune responses. Sci Signal 2010;3:pe27.

- 33 Grillo MA, Colombatto S: Metabolism and function in animal tissues of agmatine, a biogenic amine formed from arginine. Amino Acids 2004;26:3-8.

-34 Bansal V, Ochoa JB: Arginine availability, arginase, and the immune response. Curr Opin Clin Nutr Metab Care 2003; 6:223-228.

35 Masuda H: Significance of nitrogen oxide and its modulation mechanisms by endogenous nitrogen oxide synthase inhibitors and arginase in the micturition disorders and erectile dysfunction. Int J Urol 2008;15:128-134.

36 Popovic PJ, Zeh HJ III, Ochoa B: Arginine and immunity. J Nutr 2007;137:1681S-1686S.

-37 Ten Broeke R, De Crom R, Van Haperen R, Verweij V, Leusink-Muis T, Van Ark I, De Clerck F, Nijkamp FP, Folkerts G: Overexpression of endothelial nitric oxide synthase suppresses features of allergic asthma in mice. Respir Res 2006; 7:58.

- 38 Corsetti G, Stacchiotti A, D’Antona G, Nisoli E, Dioguardi FS, Rezzani R: Supplementation with essential amino acids in middle age maintains the health of rat kidney. Int J Immunopathol Pharmacol 2010;23:523-533.

- 39 Corsetti G, Pasini E, D’Antona G, Nisoli E, Flati V, Assanelli D, Dioguardi FS, Bianchi R: Morphometric changes induced by amino acid supplementation in skeletal and cardiac muscles of old mice. Am J Cardiol 2008;101S:26E-34E. 


\section{Nutrige \\ Nutrigenomics}

\begin{tabular}{l|l}
\hline J Nutrigenet Nutrigenomics 2011;4:90-98 \\
\hline $\begin{array}{l}\text { DOI: 10.1159/000327777 } \\
\text { Published online: May 28, 2011 }\end{array}$ & ๑ 2011 S. Karger AG, Basel \\
\hline Dioguardi: Arginine: To Give or Not to Give? &
\end{tabular}

-40 D’Antona G, Ragni M, Cardile A, Tedesco L, Dossena M, Bruttini F, Caliaro F, Corsetti G, Bottinelli R, Carruba MO, Valerio A, Nisoli E: Branched-chain amino acid supplementation promotes survival and supports cardiac and skeletal muscle mitochondrial biogenesis in middle-aged mice. Cell Metab 2010;12:362-372.

-41 Scognamiglio R, Negut C, Piccolotto R, Dioguardi FS, Tiengo A, Avogaro A: Effects of oral amino acid supplementation on myocardial function in patients with type 2 diabetes mellitus. Am Heart J 2004; 147:1106-1112.

-42 Dal Negro RW, Aquilani R, Bertacco S, Boschi F, Micheletto C, Tognella S: Comprehensive effects of supplemented essential amino acids in patients with severe COPD and sarcopenia. Monaldi Arch Chest Dis 2010;73:25-33.

-43 Solerte SB, Fioravanti M, Locatelli E, Bonacasa R, Zamboni M, Basso C, Mazzoleni A, Mansi V, Geroutis N, Gazzaruso C: Improvement of blood glucose control and insulin sensitivity during a long-term ( 60 weeks) randomized study with amino acid dietary supplements in elderly subjects with type 2 diabetes mellitus. Am J Cardiol 2008;101:82E$88 \mathrm{E}$.

44 Laviano A, Aghilone F, Colagiovanni D, Fiandra F, Giambarresi R, Tordiglione P, Molfino A, Muscaritoli M, Rosa G, Rossi Fanelli F: Metabolic and clinical effects of the supplementation of a functional mixture of amino acids in critically ill patients: a pilot study. Neurocrit Care, DOI: 10.1007/s12028-010-9461-Z.

-45 Pasini E, Flati V, Paiardi S, Rizzoni D, Porteri E, Aquilani R, Assanelli D, Corsetti G, Speca S, Rezzani R, DeCiuceis C, Agabiti-Rosei E: Intracellular molecular effects of insulin resistance in patients with metabolic syndrome. Cardiovasc Diabetol 2010;9:46.

46 Aquilani R, Opasich C, Dossena M, Iadalrola A, Gualco A, Arcidiaco P, Viglio S, Boschi F, Verri M, Pasini E: Increased skeletal muscle amino acid release with light exercise in deconditioned patients with heart failure. J Am Coll Cardiol 2005; $45: 158-160$. Layman DK: Dietary Guidelines should reflect new understandings about adult protein needs. Nutr Metab 2009;6:12. Scarabelli TM, Pasini E, Stephanou A, Chen-Scarabelli C, Saravolatz L, Knight RA, Latchman DS, Gardin JM: Nutritional supplementation with mixed essential amino acids enhances myocyte survival, preserving mitochondrial functional capacity during ischemia-reperfusion injury. Am J Cardiol 2004;93:35A-40A.

49 Aquilani R, Zuccarelli GC, Dioguardi FS, Baiardi P, Frustaglia A, Rutili C, Comi E, Catani M, Iadarola P, Viglio S, Barbieri A, D’Agostino L, Verri M, Pasini E, Boschi F: Effects of oral amino acid supplementation on long-term-careacquired infections in elderly patients. Arch Gerontol Geriatr, DOI: 10.1016/j.archger.2010.09.005. 